\title{
MOLECULAR CLOUDS OBSERVED WITH THE EGRET GAMMA-RAY TELESCOPE
}

\author{
SETH. W. DIGEL, STANLEY D. HUNTER, \\ AND RESHMI MUKHERJEE \\ NASA/Goddard Space Flight Center \\ EUGÉNE J. DE GEUS \\ California Institute of Technology \\ ISABELLE A. GRENIER \\ Centre d'Etudes Saclay \\ ANDREAS HEITHAUSEN \\ Universität zu Köln \\ GOTTFRIED KANBACH \\ Max-Planck-Institut für Extraterrestrische Physik \\ AND \\ PATRICK THADDEUS \\ Harvard-Smithsonian Center for Astrophysics
}

\section{Introduction}

EGRET, the high-energy $\gamma$-ray telescope on the Compton Gamma-Ray Observatory, has the sensitivity, angular resolution, and background rejection necessary to study diffuse $\gamma$-ray emission from the interstellar medium (ISM). High-energy $\gamma$ rays produced in cosmic-ray (CR) interactions in the ISM can be used to determine the CR density and calibrate the CO line as a tracer of molecular mass. Dominant production mechanisms for $\gamma$ rays of energies $\sim 30 \mathrm{MeV}-30 \mathrm{GeV}$ are the decay of pions produced in collisions of CR protons with ambient matter and Bremsstrahlung scattering of CR electrons.

Studies of diffuse $\gamma$-ray emission with EGRET are perhaps the best way to derive $X=N\left(\mathrm{H}_{2}\right) / W_{\mathrm{CO}}$. Methods for determining $X$ require indirect determinations of molecular column density, such as dust optical depth measurements or virial mass calculations. These methods require assump- 
tions, such as single, uniform dust temperatures, or virialized clouds, often of questionable validity. The assumptions involved in adopting $\gamma$-ray intensity as a tracer of column density, that high-energy CRs uniformly penetrate atomic and molecular gas and that molecular clouds do not contain $\gamma$-ray sources distributed like the molecular gas, are less serious and more widely applicable. The validity of the latter assumption cannot be proved, but at the resolution of EGRET, observational evidence indicates that embedded sources in nearby molecular clouds would have to be distributed like the gas on the scale of a few parsecs to be unresolved; such sources would be too numerous to be understood.

Interstellar cloud complexes best suited for study with EGRET are nearby, therefore subtending large solid angles, and either far from the plane or toward the outer Galaxy, limiting confusion from diffuse emission at greater distances. To date, the Ophiuchus (Hunter et al. 1994), Orion (Digel, Hunter, and Mukherjee 1995a), and Cepheus Flare complexes, as well as the complexes behind the Flare in the Perseus arm (Digel et al. 1995b), have been studied. A summary of the findings is presented here; the reader is referred to the references cited for important details of the analysis, which is founded on maximum likelihood analyses of diffuse emission with COS-B (e.g., Bloemen et al. 1984; Strong et al. 1988; Grenier and Lebrun 1990), and discussions of the $\gamma$-ray emissivities and CR densities.

\section{Results and Discussion}

Table 1 compares the values of $X$ determined by various methods for the molecular clouds studied with EGRET. The EGRET findings, all for energies $E>100 \mathrm{MeV}$, are in general agreement with non- $\gamma$-ray determinations. The origin of the difference with the COS-B study of Orion by Bloemen et al. (1984) is not clear; however, the model used for COS-B's instrumental background had not been finalized at the time of that study. For COS-B it is an important consideration; the instrumental background intensity is at least as great as that due to the molecular gas, has structure on about the same angular scale, and if incompletely subtracted would increase the value of $X$ derived. The disagreement with the COS-B study of Cepheus is less serious and may be due to the presence of two $\gamma$-ray point sources included in the EGRET analysis that were not resolved by COS-B. The near-agreement of $X$ for the three regions is remarkable on consideration of their large range of masses and different star-formation histories; the best average value for local clouds is $X=(1.0 \pm 0.2) \times 10^{20}$ molecules $\mathrm{cm}^{-2}(\mathrm{~K}$ $\left.\mathrm{km} \mathrm{s}^{-1}\right)^{-1}$, on scales of $\sim 3 \mathrm{pc}$ and larger.

The EGRET results suggest that $X$ in the Perseus arm may be greater than at the Solar circle. A trend of increasing $X$ with Galactocentric dis- 
Table 1. Determinations of the $X$-ratio

\begin{tabular}{llcl}
\hline & Method & $X^{a}$ & \\
\hline Ophiuchus & Opt. extinction & $1.1 \pm 0.5$ & Lebrun \& Huang (1984) \\
& Dust opt. depth & $>0.5_{-0.2}^{+0.5}$ & de Geus \& Burton (1991) \\
& EGRET & $1.1 \pm 0.2$ & Hunter et al. (1994) \\
Orion & COS-B & $2.2 \pm 0.6$ & Bloemen et al. (1984) \\
& ${ }^{13}$ CO/ ${ }^{12}$ CO LTE & $\sim 0.9$ & Maddalena et al. (1986) \\
& EGRET & $1.06 \pm 0.14$ & Digel et al. (1995a) \\
& COS-B & $1.9 \pm 1.0$ & Grenier \& Lebrun (1990) \\
\multirow{5}{*}{ Outer Galaxy } & CGRET $(R \sim 12-13 \mathrm{kpc})$ & $0.92 \pm 0.14$ & Digel et al. (1995b) \\
& CO (12-13 kpc) & $8 \pm 4$ & Mead \& Kutner (1988) \\
& CO $(\sim 11 \mathrm{kpc})$ & $4-6$ & Sodgel et al. (1990) \\
& EGRET $(\sim 11 \mathrm{kpc})$ & $2.5 \pm 0.9$ & Digel et al. (1995b) \\
\hline
\end{tabular}

${ }^{a}$ Units: $10^{20}$ molecules $\mathrm{cm}^{-2}\left(\mathrm{~K} \mathrm{~km} \mathrm{~s}^{-1}\right)^{-1}$; scaled to the corrected $\mathrm{CO}$ radiation temperature scale of Bronfman et al. (1988) if necessary.

tance in the outer Galaxy has been suggested by several CO-only studies (Table 1). The segment of the Perseus arm behind the Cepheus flare contains the most massive molecular clouds in the Perseus arm, the complexes associated with Cas A and NGC 7538. That the $\gamma$-ray $X$-ratio is only marginally determined even there suggests that a far larger segment of the outer Galaxy will have to be studied in order to significantly reduce the uncertainty.

\section{References}

Bloemen, J. B. G. M., Caraveo, P. A., Hermsen, W., Lebrun, F., Maddalena, R. J., Strong, A. W., Thaddeus, P. 1984, $A \& A, 139,37$

Bronfman, L., Cohen, R. S., Alvarez, H., May, J., Thaddeus, P. 1988, ApJ, 324, 248

de Geus, E. J., Burton, W. B. 1991, $A \& A$, 246, 559

Digel, S., Bally, J., Thaddeus, P. 1990, $A p J$, 357, L29

Digel, S. W., Hunter, S. D., Mukherjee, R. 1995a, $A p J$, 441, 270

Digel, S. W., Grenier, I. A., Heithausen, A., Hunter, S. D., Thaddeus, P. 1995b, ApJ, submitted

Grenier, I. A., Lebrun, F. 1990, ApJ, 360, 129

Hunter, S. D., Digel, S. W., de Geus, E. J., Kanbach G. 1994, ApJ, 436,216

Lebrun, F., Huang, Y. 1984, ApJ, 281, 634

Maddalena, R. J., Morris, M., Moscowitz, J., Thaddeus, P. 1986, ApJ, 303, 375

Mead, K. N., Kutner, M. L. 1988, ApJ, 330, 399

Sodroski, T. J. 1991, ApJ, 366, 95

Strong, A. W., et al. 1988, $A \& A, 207,1$ 